\title{
Experiencia de una Unidad de Ventilación Mecánica Pediátrica Prolongada en un hospital público de Chile
}

\author{
Experience in a Pediatric Prolonged Mechanical Ventilation \\ Unit from a public hospital in Chile
}

\author{
Dr. Daniel Zenteno ${ }^{a, b}$, Klgo. MSc. Gerardo Torres-Puebla ${ }^{a, c}$, Klga. Ximena Navarro ${ }^{a}$, \\ Enf. Univ. Carla Rivas ${ }^{a}$ Dr. Jaime Tapia ${ }^{a}$, Klgo. Dr. Iván Rodríguez-Núñez ${ }^{c}$ y \\ Dr. Javier Cepeda ${ }^{a}$
}

\section{RESUMEN}

Introducción: Los pacientes hospitalizados con altas dependencias tecnológicas respiratorias son cada vez más frecuentes y generan largas estadías en unidades de cuidados intensivos. Las estrategias que mitiguen su impacto han sido escasamente descritas.

Objetivo: Describir 6 años de experiencia de una Unidad de Ventilación Mecánica Prolongada Pediátrica.

Métodos: Estudio retrospectivo. Se incluyeron todos los niños ingresados a la Unidad entre 10-2012 y 12-2018. Se realizó estadística descriptiva e inferencial, analizando tiempos de hospitalización y reingresos. Se compararon distintas variables según tipo de patología y ventilación mecánica.

Unidad de

Mecánica

Prolongada, Servicio de Pediatría, Hospital Guillermo Grant Benavente, Concepción, Chile.

b. Departamento de Pediatría, Facultad de Medicina, Universidad de Concepción, Concepción, Chile.

c. Departamento de Kinesiología, Facultad de Medicina, Universidad de Concepción, Concepción, Chile.

Correspondencia: Dr. Daniel Zenteno Araos:

danielzenteno@gmail. com

Financiamiento:

Ninguno.

Conflicto de intereses: Ninguno que declarar.

Recibido: $18-5-2020$

Aceptado: 30-7-2020
Resultados:113 pacientes registraron310ingresos a la Unidad. Edad de ingreso: 2,2 años (0,6-8,8); varones: el 60,2 \%. Patologías: enfermedad neuromuscular (el 22,1\%), enfermedad pulmonar crónica (el 20,4\%), daño neurológico (el 34,5\%), obstrucción de vía aérea superior (el 9,7\%), cardiopatía (el 3,5\%), síndrome de Down (el 9,7\%). Se utilizaron 10507 días / cama; con índice ocupacional del $92,6 \%$, el $54,8 \%$ de traslados a la Unidad de Cuidados Intensivos y el $66,1 \%$ de reingresos. Hospitalización media: 16 días (6,5-49,0); diferencias en edad de ingreso según patologías $(\mathrm{p}=0,032)$. Hubo más reingresos en niños con daño neurológico y síndrome de Down $(p=0,004)$. Los niños con asistencia ventilatoria invasiva presentaron más días de hospitalización $(\mathrm{p}<0,001)$ y reingresos $(\mathrm{p}<0,001)$. Conclusión: Elíndice ocupacional fue superior al $90 \%$; permitió mayor disponibilidad de camas intensivas y egresar a todos los pacientes. Los niños con asistencia ventilatoria invasiva se hospitalizaron más tiempo y reingresaron más. Palabras clave: ventilación mecánica, atención domiciliaria de salud, enfermedades respiratorias crónicas, traqueostomía, pediatría.

http: / / dx.doi.org/10.5546/ aap.2021.25

Texto completo en inglés:

http: / / dx.doi.org/ 10.5546/ aap.2021.eng.25

Cómo citar: Zenteno D, Torres-Puebla G, Navarro X, Rivas C, et al. Experiencia de una Unidad de Ventilación Mecánica Pediátrica Prolongada en un hospital público de Chile. Arch Argent Pediatr 2021;119(1):25-31.

\section{GLOSARIO}

AVI: asistencia ventilatoria invasiva. AVNI: asistencia ventilatoria no invasiva.

DN: daño neurológico.

ENM: enfermedad neuromuscular. EPC: enfermedad pulmonar crónica.

ERC: enfermedad respiratoria crónica. OVAS: obstrucción de la vía aérea superior.

SD: síndrome de Down.

UCIP: Unidad de Cuidados

Intensivos Pediátricos.

UVMP: Unidad de Ventilación

Mecánica Prolongada.

\section{INTRODUCCIÓN}

Los avances en unidades de cuidados intensivos pediátricos y neonatales, la utilización de esquemas terapéuticos más efectivos y el desarrollo de programas focalizados de intervención en salud infantil han permitido incrementar la supervivencia de niños con enfermedades respiratorias crónicas (ERC). Ellos requieren de recursos tecnológicos y un alto nivel de especialización profesional para un adecuado diagnóstico, tratamiento y seguimiento de su enfermedad. ${ }^{1,2}$

Esta realidad propicia la hospitalización de dichos pacientes en unidades de alta complejidad, como las unidades de cuidados intensivos pediátricos (UCIP), con estadías prolongadas, lo que reduce la rotación de pacientes agudos complejos en estado crítico, en la red asistencial, y eleva el costo directo y de oportunidad de la atención. ${ }^{3}$ 
Constituye un desafío para los modelos de atención en salud implementar alternativas innovadoras y eficaces para el manejo hospitalario de estos pacientes con ERC. ${ }^{4,5} \mathrm{Un}$ alto porcentaje de estos niños son subsidiarios de ventilación mecánica prolongada, la que se define como la necesidad de soporte ventilatorio invasivo o no invasivo por un período mayor de 21 días, por más de 6 horas al día y con interrupciones inferiores a 48 horas. ${ }^{6}$

La experiencia reportada, tanto a nivel local como internacional, sugiere que la terapia ventilatoria puede iniciarse en el hospital y continuarse en el domicilio del niño. Esto obliga a reducir la probabilidad de eventos adversos, así como a asegurar la inclusión social del niño y su familia, lo cual supone la necesidad de equipamiento tecnológico, personal capacitado, instrucción y seguimiento a los padres y cuidadores de los pacientes subsidiarios.,

En Chile, la prevalencia de niños con ERC se ha incrementado significativamente en los últimos 20 años, lo que ha motivado la creación de programas ministeriales destinados al tratamiento, rehabilitación y apoyo de las necesidades tecnológicas especiales ${ }^{8-10}$ En este contexto, se creó, el año 2012, la Unidad de Ventilación Mecánica Prolongada (UVMP), con 5 camas en el Servicio de Pediatría del Hospital Guillermo Grant Benavente de la ciudad de Concepción. Desde su desarrollo, se orientó a dar atención multidisciplinaria a niños con ERC complejas por un equipo multidisciplinario integrado por enfermeras y kinesiólogos del staff; apoyados por fonoaudiólogos, terapeutas ocupacionales, trabajadores sociales, psicóloga y coordinado por un pediatra broncopulmonar. Este solicita evaluaciones periódicas de múltiples especialidades médicas (otorrinolaringólogo, neurólogo, cardiólogo, nutriólogo, endocrinólogo, oftalmólogo y cirujanos), lo que permite la transición entre la UCIP y el hogar, donde se entregan herramientas de rehabilitación clínica y educativas que persiguen logran un egreso hospitalario seguro y efectivo. ${ }^{3,11}$ Las reagudizaciones más complejas que generan inestabilidad clínica son derivadas a la UCIP.

El objetivo de este estudio fue describir la experiencia de la UVMP del Servicio de Pediatría del Hospital Guillermo Grant Benavente, las características de los pacientes ingresados y sus reingresos, luego de 6 años de funcionamiento.

\section{MÉTODOS}

El estudio es una serie de casos retrospectiva, redactado de acuerdo con la declaración Strengthening the Reporting of Observational Studies in Epidemiology (STROBE). ${ }^{12}$ Se incluyeron todos los niños ingresados a la UVMP desde su apertura (octubre de 2012) hasta diciembre de 2018. En la Figura 1, se observa el diagrama de ingresos, egresos y reingresos a la UVMP. No se consideraron criterios de exclusión.

El motivo de ingreso a la UVMP fue categorizado de la siguiente manera: (a) inicio de ventilación mecánica: pacientes que ingresaron para evaluar la necesidad de asistencia ventilatoria invasiva (AVI) o asistencia ventilatoria no invasiva (AVNI) domiciliaria y recibir educación en oxigenoterapia, aerosolterapia, cuidados de ventilación mecánica y vía aérea artificial, ejercicios respiratorios y reanimación cardiopulmonar básica, por un equipo multidisciplinario a través de folletos, manuales y actividades teórico-prácticas por personal capacitado; (b) reevaluación multidisciplinaria programada: pacientes que ingresaron para evaluación por múltiples especialidades; (c) posreagudización: pacientes clínicamente estables que ingresaron después de un cuadro de reagudización de origen respiratorio o no respiratorio desde unidades críticas o urgencias; (d) proceso de decanulación: pacientes traqueostomizados que ingresaron para comenzar o adecuar su planificación de decanulación; (e) procedimiento médico-quirúrgico: pacientes crónicos respiratorios que ingresaron para procedimientos médico-quirúrgicos sin cumplir los motivos anteriores.

Al ingresar a la UVMP, se registraron las variables demográficas de los niños, como edad, sexo, diagnóstico, unidad de procedencia, tipo de ventilación mecánica (AVI, AVNI), causa de ingreso y días de estadía hospitalaria. El diagnóstico de ingreso se clasificó en enfermedad neuromuscular (ENM), enfermedad pulmonar crónica (EPC), daño neurológico central (DN), obstrucción de la vía aérea superior (OVAS), cardiopatía y síndrome de Down (SD).

\section{Análisis estadístico}

Se realizó el análisis de los datos con el test de normalidad Kolmogorov-Smirnov. Se descartó la distribución normal, por lo que se reportó mediana y rango intercuartílico para variables cuantitativas, porcentaje para variables cualitativas, y se usó el test de Kruskal-Wallis para 
comparar los días de hospitalización, el número de ingresos y los reingresos según el diagnóstico y el tipo de ventilación mecánica. Adicionalmente, se determinó el índice ocupacional o porcentaje de ocupación, según la relación entre el número total de días / camas ocupadas durante un período y el total de camas disponibles en el período, expresado en porcentaje. ${ }^{8}$

El análisis se realizó con el software IBM SPSS Statistics ${ }^{\circledR}$ para Windows, v.23.0 (IBM Corporation. Armonk, Nueva York, Estados Unidos) y se consideró significativo un valor de $p<0,05$. El estudio fue aprobado por el Comité Ético de la Universidad de Concepción. Los datos fueron ingresados a una planilla sin antecedentes personales de identificación, codificados, y el archivo fue protegido por contraseña.

\section{RESULTADOS}

Durante los 6 años considerados, ingresaron 113 niños a la UVMP con una edad de ingreso de 2,2 años (0,6-8,8); el 60,2 \% eran varones (Tabla 1). El 22,1\% de la muestra correspondió a individuos con ENM; el 20,4 \%, con EPC; el 34,5\%, con DN; el 9,7\%, con OVAS; el 3,5\%, con cardiopatía, y el $9,7 \%$, con SD (Tabla 1).

De los 113 niños ingresados, el 87,6 \% fue evaluado para el inicio de ventilación mecánica $\mathrm{y}$, finalmente, el 61,9\% del total requirió manejo con soporte ventilatorio prolongado,

Figura 1. Diagrama de flujo de ingresos, egresos y reingresos a la Unidad de Ventilación Mecánica Prolongada

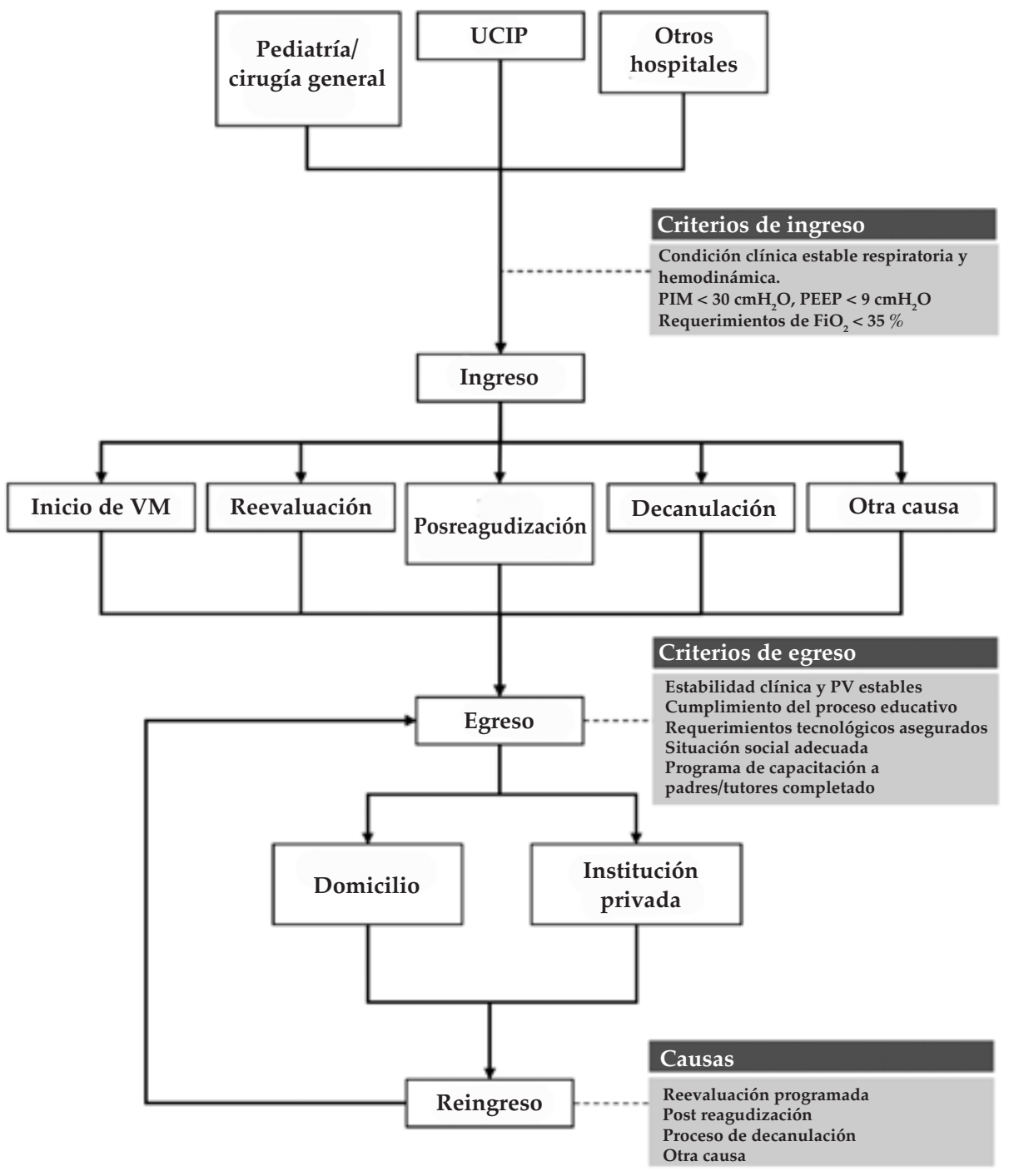


en modalidad invasiva y no invasiva. Del total, 38 niños solo ingresaron para la evaluación inicial, sin reingresos posteriores. Los 75 niños restantes reingresaron por diversas causas: 36 por reevaluación, 56 por reagudización, 6 para planificar la decanulación y 9 por procedimientos médico-quirúrgicos (Tabla 2).

Las 5 camas de la UVMP permitieron 310 ingresos en el período, que ocuparon 10507 días / cama, con un índice ocupacional global del 92,6\%. De ellos, el 46,1 \% provenían directamente de camas críticas (intermedio o intensivo), quienes ocuparon 8577 días/camas, con estadía de 17 días (7-44). Del total de ingresos, el 63,9\% correspondió a reingresos, de los cuales el 73,2 \% fue posreagudización y, de estos, el $84,8 \%$ por causa respiratoria y el $15,2 \%$ por otra causa. El 38,9 \% de los pacientes reingresaron durante el primer año de egreso.

La totalidad de la muestra presentó diferencias en la edad de ingreso a la UVMP; los menores fueron aquellos con OVAS, y los mayores, con ENM ( $p=0,032)$. Los niños con DN y SD presentaron más días de hospitalización respecto a las otras categorías; mientras que aquellos con ENM permanecieron menos días hospitalizados $(\mathrm{p}=0,169)$ (Tabla 1$)$.

Los sujetos con DN y SD presentaron una mediana de 2 reingresos durante el período analizado $(p=0,004)$; mientras que los mayores reingresos posreagudización fueron en niños con EPC, DN y OVAS, con una mediana de 1 $(p=0,046)$. En la Tabla 2, se observan los niños ingresados y reingresados según el subgrupo de patologías.

Dependiendo del tipo de ventilación mecánica utilizada, existieron diferencias entre los grupos en días de hospitalización $(\mathrm{p}<0,001)$, el número de reingresos $(\mathrm{p}<0,001)$, el número de reevaluaciones $(\mathrm{p}=0,001)$, los reingresos posreagudización $(\mathrm{p}<0,001)$ y por procedimiento médico-quirúrgico $(\mathrm{p}=0,018)$, y los niños con AVI fueron quienes presentaron mayor cantidad de días de hospitalización y reingresos que los otros grupos (Tabla 3). El $18 \%$ de este grupo tuvo un proceso de decanulación exitoso.

En los niños con AVNI, las hospitalizaciones posreagudización fueron menos frecuentes

TABLA 1. Características generales de la muestra total y por subgrupos de patologías durante el primer ingreso, expresadas en mediana y rango intercuartílico

\begin{tabular}{|c|c|c|c|c|c|c|c|}
\hline Variables & $\begin{array}{c}\text { Total } \\
(n=113)\end{array}$ & $\begin{array}{c}\text { ENM } \\
(n=25)\end{array}$ & $\begin{array}{c}\text { EPC } \\
(n=23)\end{array}$ & $\begin{array}{c}\text { DN } \\
(n=39)\end{array}$ & $\begin{array}{l}\text { OVAS } \\
(\mathrm{n}=11)\end{array}$ & $\begin{array}{c}\text { Cardiopatía } \\
(n=4)\end{array}$ & $\begin{array}{c}\text { SD } \\
(\mathrm{n}=11)\end{array}$ \\
\hline $\begin{array}{l}\text { Edad al momento } \\
\text { del ingreso (años) }\end{array}$ & $\begin{array}{c}2,2 \\
(0,6-8,8)\end{array}$ & $\begin{array}{c}5,5 \\
(1,4-12,6)\end{array}$ & $\begin{array}{c}0,8 \\
(0,5-12,4)\end{array}$ & $\begin{array}{c}4,6 \\
(1,2-9,5)\end{array}$ & $\begin{array}{c}0,7 \\
(0,3-1,0)\end{array}$ & $\begin{array}{c}0,9 \\
(0,3-3,0)\end{array}$ & $\begin{array}{c}1,5 \\
(0,6-2,2)\end{array}$ \\
\hline Sexo (M/F) & $68 / 45$ & $14 / 11$ & $18 / 5$ & $19 / 20$ & $8 / 3$ & $2 / 2$ & $7 / 4$ \\
\hline $\begin{array}{l}\text { Ventilación } \\
\text { mecánica } \\
\text { (AVI/AVNI) }\end{array}$ & $33 / 37$ & $7 / 13$ & $4 / 5$ & $13 / 14$ & $4 / 3$ & $0 / 1$ & $5 / 1$ \\
\hline $\begin{array}{l}\text { Estancia } \\
\text { hospitalaria } \\
\text { (días) }\end{array}$ & $\begin{array}{c}16,0 \\
(6,5-49,0)\end{array}$ & $\begin{array}{c}7,0 \\
(3,0-63,5)\end{array}$ & $\begin{array}{c}14,0 \\
(6,0-35,0)\end{array}$ & $\begin{array}{c}30,0 \\
(10,0-63,0)\end{array}$ & $\begin{array}{c}13,0 \\
(6,0-61,0)\end{array}$ & $\begin{array}{c}9,5 \\
(5,3-14,5)\end{array}$ & $\begin{array}{c}20,0 \\
(13,0-79,0)\end{array}$ \\
\hline
\end{tabular}

ENM: enfermedad neuromuscular; EPC: enfermedad pulmonar crónica; DN: daño neurológico; OVAS: obstrucción de la vía aérea superior; SD: síndrome de Down; M: masculino; F: femenino; AVI: asistencia ventilatoria invasiva; AVNI: asistencia ventilatoria no invasiva.

TABLA 2. Número de pacientes ingresados según la causa por grupo total y subgrupos de patologías

\begin{tabular}{lccc}
\hline Diagnóstico & Inicio de VM & Reevaluación & Posreagudización \\
\hline SD $(\mathrm{n}=11)$ & 11 & 6 & 5 \\
Cardiopatía $(\mathrm{n}=4)$ & 4 & 0 & 1 \\
OVAS (n=11) & 11 & 4 & 6 \\
DN $(\mathrm{n}=39)$ & 36 & 15 & 23 \\
EPC $(\mathrm{n}=23)$ & 17 & 4 & 13 \\
ENM $(\mathrm{n}=25)$ & 20 & 7 & 8 \\
Total $(\mathrm{n}=113)$ & 99 & 36 & 56 \\
\hline
\end{tabular}

VM: ventilación mecánica; SD: síndrome de Down; OVAS: obstrucción de la vía aérea superior; DN: daño neurológico; EPC: enfermedad pulmonar crónica; ENM: enfermedad neuromuscular. 
y obedecieron, principalmente, a causas respiratorias en el 79,7 \% de los ingresos. Las hospitalizaciones electivas se relacionaban con procedimientos más invasivos, como necesidad de gastrostomía (el 1,6\%) o necesidades de reevaluaciones multidisciplinarias, frente a casos complejos y difíciles de evaluar ambulatoriamente (el 16,4\%).

El $99 \%$ de los niños fue egresado del Hospital a su domicilio o institución privada en los casos con condicionantes sociales y, de los pacientes que requirieron soporte ventilatorio domiciliario al egresar, el 47,1 \% correspondió a AVI, y el 52,9\%, a AVNI. El tiempo de hospitalización fue de 16,0 días (6,5-49,0). Solo 1 niño con AVI falleció en la UVMP, que fue manejado con cuidados paliativos a causa del avanzado estado de su patología de base.

\section{DISCUSIÓN}

El presente artículo reporta 6 años de experiencia de una UVMP en un hospital público de Chile, que incluyó estrategias respiratorias de estudio y tratamiento, rehabilitación individualizada y educación dirigida a padres y cuidadores. Durante el período estudiado, se registraron 310 ingresos en 113 usuarios, que utilizaron un total de 10507 días de hospitalización, con un índice ocupacional $>90 \%$ en la UVMP. Esto permitió, consecuentemente, una mayor disponibilidad de cupos en la UCIP para pacientes agudos críticos y evitó estadías prolongadas de pacientes con altas dependencias tecnológicas respiratorias crónicas en dicha Unidad.

Nuestros resultados concordaron con los de Paulides y cols., quienes documentaron también hospitalizaciones prolongadas en este grupo de pacientes, que limitaron la disponibilidad de cupos de camas críticas pediátricas. En este reporte, comunicaron su experiencia de 30 años, con casi 300 niños dependientes de soporte ventilatorio, quienes fueron hospitalizados durante 12440 días antes de su egreso domiciliario y requirieron 9335 días de hospitalización, por reingresos graves a la UCIP. ${ }^{3}$

En un reporte anterior de nuestro grupo, se constató que la implementación de esta Unidad permitió aumentar los índices de rotación y reducir los tiempos de la estadía en la UCIP significativamente. ${ }^{11}$ Esto concordó con Amirnovin y cols., quienes reportaron una disminución del promedio de días de estadía en la UCIP de 70 a 36 con la implementación de una estrategia hospitalaria similar. ${ }^{5}$

Los diagnósticos más frecuentes fueron ENM, EPC, DN y OVAS; con una edad de ingreso significativamente menor en los pacientes con OVAS y mayor en aquellos con ENM. En el caso de los OVAS, esto tuvo relación con la gravedad de los niños a inicios tempranos de la vida, ya que, en nuestra cohorte de niños ingresados con OVAS menores de un año, un $78 \%$ requirió traqueostomía y/o soporte ventilatorio a su alta domiciliaria, tal como fue descrito previamente en casos graves. Sin embargo, muchas veces, su resolución final se establece mediante cirugía de avance maxilar o mandibular. ${ }^{13}$ La distracción mandibular se realizó en el $33 \%$ de nuestros pacientes con OVAS menores de un año, con el fin de evitar la traqueostomía, situación que fue reportada previamente en menores con hipoplasia mandibular grave, y demostró su eficacia, seguridad y evitó dependencias respiratorias. ${ }^{14,15}$ Para optimizar el manejo ventilatorio en aquellos con OVAS menores de un año, el $22 \%$ utilizó AVNI, lo que evitó la intubación y la traqueostomía, tal como mostraron Leboulanger y cols., en una cohorte de recién nacidos con secuencia de Pierre Robin, en la que propusieron considerar este tipo de soporte como una línea inicial de tratamiento. ${ }^{16}$

Tabla 3. Días de hospitalización, número de reingresos y causas de reingreso según el tipo de ventilación mecánica

\begin{tabular}{lcccccc}
\hline $\begin{array}{l}\text { Tipo de ventilación } \\
\text { mecánica }\end{array}$ & $\begin{array}{c}\text { Estancia hospitalaria } \\
\text { Días }\end{array}$ & $\mathbf{n}$ & $\begin{array}{c}\text { Reingreso } \\
\mathbf{N}^{\circ} \text { de ingresos }\end{array}$ & $\mathbf{n}$ & $\begin{array}{c}\text { Reevaluación } \\
\mathbf{N}^{\circ} \text { de ingresos }\end{array}$ & $\begin{array}{c}\text { Posreagudización } \\
\mathbf{n} \mathbf{N}^{\circ} \text { de ingresos }\end{array}$ \\
\hline $\begin{array}{l}\text { AVI } \\
(\mathbf{n}=33)\end{array}$ & $\begin{array}{c}61,0 \\
(14,0-179,5)^{\mathrm{ab}}\end{array}$ & 21 & 73 & 11 & 15 & 18 \\
$\begin{array}{l}\text { AVNI } \\
(\mathbf{n}=37)\end{array}$ & $\begin{array}{c}14,0 \\
(5,0-35,8)^{\mathrm{a}}\end{array}$ & 17 & 66 & 12 & 16 & 16 \\
$\begin{array}{l}\text { Sin VM } \\
(\mathbf{n}=\mathbf{4 3})\end{array}$ & $\begin{array}{c}13,0 \\
(7,3-30,8)^{\mathrm{b}}\end{array}$ & 3 & 5 & 0 & 0 & 51 \\
\hline
\end{tabular}

Datos expresados en mediana y rango intercuartílico. AVI: asistencia ventilatoria invasiva; AVNI: asistencia ventilatoria no invasiva; VM: ventilación mecánica. a, b: p <0,05 entre variables evaluadas según el tipo de ventilación mecánica. 
Las ENM incluidas recibieron la indicación de AVNI domiciliaria en un $63 \%$, que correspondió, sobre todo, al grupo de ENM lentamente progresivas, que requerían cuidados respiratorios y soporte ventilatorio en una etapa posterior de la vida. ${ }^{17,18}$ Hoy existe consenso en cuanto a la necesidad de iniciar precozmente la ventilación no invasiva en pacientes con enfermedades neuromusculares progresivas, debido al impacto positivo en el curso clínico de estas enfermedades. ${ }^{17,18} \mathrm{Si}$ bien la necesidad de soporte ventilatorio debe ser observando un trastorno respiratorio del sueño, frecuentemente, esta terapia se inicia luego de una hospitalización por causa respiratoria. ${ }^{18,19}$ Esta situación ha sido reportada en la literatura y dependerá del tipo de enfermedad neuromuscular, de los tratamientos disponibles y otros factores pronósticos relacionados. ${ }^{18,20}$

La implementación de estrategias que permiten otorgar un soporte ventilatorio domiciliario invasivo y no invasivo ha sido utilizada en varios países con un categórico aumento durante los 30 últimos años y ha demostrado sus resultados favorables para los pacientes y los sistemas sanitarios. ${ }^{3,4,11,21,22}$ En nuestro grupo de niños, quienes cumplieron con el respaldo socioeconómico domiciliario ingresaron a programas ministeriales implementados en Chile desde 2006 para AVNI y desde 2008 para AVI, los cuales contaban con 658 pacientes pediátricos activos y 1212 ingresados, hasta inicios del 2019. ${ }^{8-10,23}$ Para el grupo de pacientes sin condiciones socioeconómicas requeridas por estos programas para regresar con asistencia ventilatoria a domicilio, se implementó un equipo técnico y profesional en una institución externa al recinto hospitalario. . $^{8-10,24}$

El $54 \%$ del universo de nuestros niños requirió el reingreso no programado a la UVMP, la mayor parte con AVI, lo cual puede explicarse por la mayor complejidad de sus patologías de base, sus complicaciones asociadas y la necesidad de evaluaciones periódicas para determinar conductas que puedan implicar un avance $o$ regresión de su patología de base. ${ }^{25-27}$ Kun y cols., describieron los reingresos no programados en pacientes con AVI en domicilio y reportaron un $40 \%$ de reingresos a los 12 meses del egreso hospitalario, de los cuales el $64 \%$ se relacionaban con causas respiratorias. ${ }^{25}$ En nuestro grupo de niños con AVI en domicilio, los resultados fueron similares: el $30 \%$ fueron reingresados de forma no programada dentro del primer año, y el $67 \%$ de estos, por causa respiratoria.

Los reingresos programados también fueron más frecuentes en niños con AVI, que, generalmente, poseían limitación para realizar múltiples controles y exámenes. Se realizaron planes individualizados, conforme a la clínica y problemas específicos. Se incluyeron, principalmente, evaluación con especialistas, evaluaciones de la vía aérea, estudios de sueño y exámenes generales. Henningfeld y cols., describieron el proceso de destete ventilatorio y la decanulación de 46 niños con AVI, que implicaron hospitalizaciones y exámenes para su planificación y mayor índice de éxito. ${ }^{27,28}$ Esto se logró en 6 de nuestros niños que utilizaron AVI en un período inferior a 3 años desde la instalación de la traqueostomía, y fue más frecuente la liberación del ventilador antes de los 5 años del inicio de esta. ${ }^{28}$

Existen ciertas limitaciones en nuestro estudio, tales como la diversidad de las patologías categorizadas y su tamaño muestral, lo que limita las comparaciones entre estas. El estudio se realizó solo en nuestra Institución, lo cual nos permitió mostrar únicamente la realidad local con una cantidad limitada de participantes, que pudo influir en los resultados de nuestros análisis. Sin embargo, no existen reportes hasta la fecha de la realidad nacional y son escasos a nivel internacional los de este tipo de dependencias asistenciales. Además, no se incluyeron como posible factor de confusión la gravedad de las comorbilidades y las características socioeconómicas de nuestra población.

\section{CONCLUSIÓN}

Los resultados de este estudio permiten concluir que los reingresos no programados son frecuentes en nuestra UVMP; estos últimos, sobre todo, de causa respiratoria y relacionados, principalmente, con AVI. Al mismo tiempo, la UVMP logró el alta hospitalaria de todos los pacientes, independiente de su grado de complejidad. A nivel local, el desarrollo de una unidad especializada en cuidados respiratorios de pacientes con altas dependencias tecnológicas respiratorias permitió una mayor disponibilidad de cupos en la UCIP para pacientes agudos críticos.

\section{Agradecimientos}

A todo el personal de salud dedicado a los cuidados de estos pacientes con altas dependencias respiratorias en esta unidad, 
en cuidados intensivos y en su domicilio. A sus padres y familiares, que con una luz de esperanza viven el día a día, esperando mejorar las expectativas de vida de estos niños.

\section{REFERENCIAS}

1. Elias ER, Murphy NA, Liptak GS, Adams RC, et al. Home care of children and youth with complex health care needs and technology dependencies. Pediatrics. 2012; 129(5):9961005.

2. Torres-Castro R, Zenteno D, Rodriguez-Nuñez I, Villarroel G, et al. Guías de rehabilitación respiratoria en niños con enfermedades respiratorias crónicas: actualización 2016. Neumol Pediatr. 2016; 11(3):114-31.

3. Paulides F, Plötz RB, Verweij-van den Oudenrijn LP, Van GestelJPJ, et al. Thirty years of home mechanical ventilation in children: Escalating need for pediatric intensive care beds. Intensive Care Med. 2012; 38(5):847-52.

4. Amin R, Sayal A, Syed F, Daniels C, et al. How long does it take to initiate a child on long-term invasive ventilation? Results from a Canadian pediatric home ventilation program. Can Respir J. 2015; 22(2):103-8.

5. Amirnovin R, Aghamohammadi S, Riley C, Woo MS, et al. Analysis of a pediatric home mechanical ventilator population. Respir Care. 2018; 63(5):558-64.

6. Sauthier M, Rose L, Jouvet P. Pediatric prolonged mechanical ventilation: Considerations for definitional criteria. Respir Care. 2017; 62(1):49-53.

7. Moore PE, BoyerD, $\mathrm{O}^{\prime}$ Connor MG, Baker CD, et al. Pediatric chronichomeinvasive ventilation. Ann Am Thorac Soc. 2016; 13(7):1170-2.

8. Chile. Ministerio de Salud, Subsecretaría de Redes Asistenciales, División de Atención Primario, Unidad de Salud Respiratoria. Programa de Asistencia Ventilatoria No Invasiva en Atención Primaria de Salud: Normas Técnicas. Neumonol Pediatr. 2007; 2:38-48.

9. Chile. Ministerio de Salud, Subsecretaría de Redes Asistenciales, División de Atención Primario, Unidad de Salud Respiratoria. Protocolo de Ventilación Mecánica Invasiva en APS. 2013. [Acceso: 31 de julio de 2020]. Disponibleen:https: / / respiratorio.minsal.cl/PDF/AVNI/ Progama_AVI_2013.pdf.

10. Maquilón OC, Antolini TM. Jornadas de otoño 2019 “De la UCI al domicilio". Rev Chil Enferm Respir. 2019; 35(2):93-5.

11. Galaz SN, Zenteno AD, Barraza EC, Tapia ZJ, et al. Impact of a prolonged ventilation unit in pediatric service. XI SOLANEP International Congress/XV Cystic Fibrosis Latinamerican Congress/XV Brazilian Congress of Pediatric Pulmonology. Pediatr Pulmonol.2016;51(S42):180.

12. Von Elm E, Altman D, Egger M, Pocock S, et al. The Strengthening the Reporting of Observational Studies in Epidemiology (STROBE) statement: guidelines for reporting observational studies. J Clin Epidemiol. 2008; 61(4):344-9.

13. TanHL, Kheirandish-Gozal L, AbelF, GozalD. Craniofacial syndromes and sleep-related breathing disorders. Sleep
Med Rev. 2016; 27:74-88.

14. Denny A, Amm C. New technique for airway correction in neonates with severe Pierre Robin sequence. J Pediatr. 2005; 147(1):97-101.

15. Hong P. A clinical narrative review of mandibular distraction osteogenesis in neonates with Pierre Robin sequence. Int J Pediatr Otorhinolaryngol. 2011; 75(8):985-91.

16. Leboulanger N, Picard A, Soupre V, Aubertin G, et al. Physiologic and Clinical Benefits of Noninvasive Ventilation in Infants with Pierre RobinSequence. Pediatrics. 2010; 126(5):e1056-63.

17. Birnkrant DJ, Bushby K, Bann CM, Alman BA, et al. Diagnosis and management of Duchenne muscular dystrophy, part 2: respiratory, cardiac, bone health, and orthopaedic management. Lancet Neurol. 2018; 17(4):34761.

18. Hull J, Aniapravan R, Chan E, Chatwin M, et al. British Thoracic Society guideline for respiratory management of children with neuromuscular weakness. Thorax. 2012; 67(Suppl 1):i1-40.

19. Amaddeo A, Moreau J, Frapin A, Khirani S, et al. Long term continuous positive airway pressure (CPAP) and noninvasive ventilation (NIV) in children: Initiation criteria in real life. Pediatr Pulmonol. 2016; 51(9):968-74.

20. Zenteno AD, Verbal CD, Barraza EC, Elso TMJ. Estudios de sueñoen pacientes con enfermedades neuromusculares. Neumol Pediatr. 2017; 12(2):76-80.

21. Chau SK, Yung AW, Lee SL. Long-Term Management for Ventilator-Assisted Children in Hong Kong: 2 Decades' Experience. Respir Care. 2017; 62(1):54-64.

22. Amin R, Sayal P, Syed F, Chaves A, el al. Pediatric longterm home mechanical ventilation: Twenty years of follow-up from one Canadian center. Pediatr Pulmonol. 2014; 49(8):816-24.

23. Prado AF, Salinas FP. Asistencia ventilatoria no invasiva domiciliaria en niños: Impacto inicial de un programa nacional en Chile. Rev Chil Pediatr. 2011; 82(4):289-99.

24. Carrasco D, Navarro X, Herrera C, Tapia J, et al. Ventilación mecánica prolongada en la corporación nacional de nutrición infantil (CONIN)... La Luz no se apaga. Neumol Pediatr. 2017; 12(4):A196.

25. Kun SS, Edwards JD, Davidson Ward SL, Keens TG. Hospital readmissions for newly discharged pediatric home mechanical ventilation patients. Pediatr Pulmonol. 2012; 47(4):409-14.

26. Edwards JD, Kun SS, Keens TG. Outcomes and Causes of Death in Children on Home Mechanical Ventilation via Tracheostomy: An Institutional and Literature Review. J Pediatr. 2010; 157(6):955-9.e2.

27. Henningfeld JK, Maletta K, Ren B, Richards KL, et al. Liberation from home mechanical ventilation and decannulation in children. Pediatr Pulmonol. 2016; 51(8):838-49.

28. Gurbani N, Promyothin U, Rutter M, Fenchel MC, et al. Using Polysomnography and Airway Evaluation to Predict Successful Decannulation in Children. Otolaryngol Head Neck Surg. 2015; 153(4):649-55. 